\title{
A Common Cortical Metric for Spatial, Temporal, and Social Distance
}

\author{
Carolyn Parkinson, Shari Liu, and Thalia Wheatley \\ Department of Psychological and Brain Sciences, Dartmouth College, Hanover, New Hampshire 03755
}

Distance describes more than physical space: we speak of close friends and distant relatives, and of the near future and distant past. Did these ubiquitous spatial metaphors arise in language coincidentally or did they arise because they are rooted in a common neural computation? To address this question, we used statistical pattern recognition techniques to analyze human fMRI data. First, a machine learning algorithm was trained to discriminate patterns of fMRI responses based on relative egocentric distance within trials from one distance domain (e.g., photographs of objects relatively close to or far away from the viewer in spatial distance trials). Next, we tested whether the decision boundary generated from this training could distinguish brain responses according to relative egocentric distance within each of two separate distance domains (e.g., phrases referring to the immediate or more remote future within temporal distance trials; photographs of participants' friends or acquaintances within social distance trials). This procedure was repeated using all possible combinations of distance domains for training and testing the classifier. In all cases, above-chance decoding across distance domains was possible in the right inferior parietal lobule (IPL). Furthermore, the representational similarity structure within this brain area reflected participants' own judgments of spatial distance, temporal soon-ness, and social familiarity. Thus, the right IPL may contain a parsimonious encoding of proximity to self in spatial, temporal, and social frames of reference.

Key words: egocentric distance; inferior parietal lobule; multivoxel pattern analysis; psychological distance; social cognition; temporoparietal junction

\section{Introduction}

Converging theories from cognitive linguistics, cognitive neuroscience, and social psychology suggest that different domains of psychological distance are encoded similarly. Conceptual metaphor theory (CMT; Lakoff and Johnson, 1980) suggests that we use spatial language to describe social and temporal relationships (e.g., "close friend," "distant future") because we mentally represent this information in spatial terms. Neuroscientists have suggested that, over the course of evolution, mechanisms devoted to spatial processing may have been redeployed to "plot" information in increasingly abstract (e.g., temporal, social) frames of reference (Yamazaki et al., 2009; Parkinson and Wheatley, 2013). Mounting evidence from social psychology supports these assertions and suggests an explanation for overlap in the language and brain areas used to represent social, spatial, and temporal distance. The degree to which information is removed from our current experience in time, space, or the extent to which it refers

\footnotetext{
Received May 21, 2013; revised Dec. 20, 2013; accepted Dec. 29, 2013.

Author contributions: C.P. and T.W. designed research; C.P. and S.L. performed research; C.P. analyzed data; C.P. and T.W. wrote the paper.

This research was supported in part by a McNulty grant from The Nelson A. Rockefeller Center at Dartmouth to T.W. C.P. is supported by a postgraduate scholarship from the Natural Sciences and Engineering Research Council of Canada. Wethank Edmund Chong, Sergey Fogelson, Melissa Rundle, Lisa Sprute, Peter Tse, and Jim Haxby for helpful discussion.

The authors declare no competing financial interests.

Correspondence should be addressed to Carolyn Parkinson, Department of Psychological and Brain Sciences, Dartmouth College, 6207 Moore Hall, Hanover, NH 03755. E-mail: carolyn.m.parkinson.gr@dartmouth.edu. DOI:10.1523/JNEUROSCI.2159-13.2014

Copyright $\odot 2014$ the authors $\quad 0270-6474 / 14 / 341979-09 \$ 15.00 / 0$
}

to someone else carries a common meaning with important implications for the perceiver: proximity for action and, thus, how concretely or abstractly it should be construed (Vallacher and Wegner, 1985; Liberman and Trope, 2008).

The common meaning shared across psychological distance domains is accessed automatically. Words characterizing shorter social and temporal distances are automatically associated with closer spatial locations, whereas words referring to increased social and temporal distances are associated with farther locations (Bar-Anan et al., 2007). Different domains of psychological distance also impact a range of mental phenomena similarly, from visual perception (Förster et al., 2004) to humor (McGraw et al., 2012) and moral judgment (Eyal et al., 2008). Additionally, the capacity to mentally traverse different domains of distance follows a similar developmental trajectory (Suddendorf and Corballis, 2007). Thus, converging evidence is consistent with the possibility of a common mechanism for representing different domains of egocentric distance.

If different domains of egocentric distance are conflated at some level of processing, relatively near or far distances should be represented similarly at that level of processing, regardless of domain. Previous fMRI studies suggest that judging and traversing spatial, social, and temporal distances recruit overlapping brain regions (Buckner and Carroll, 2007; Spreng et al., 2009; Yamakawa et al., 2009; Yamazaki et al., 2009; Tamir and Mitchell, 2011). However, past studies have used univariate analyses that spatially smooth and average data to identify areas involved in tasks (Friston et al., 1995). Importantly, a common encoding cannot be inferred from independent univariate fMRI contrasts. 

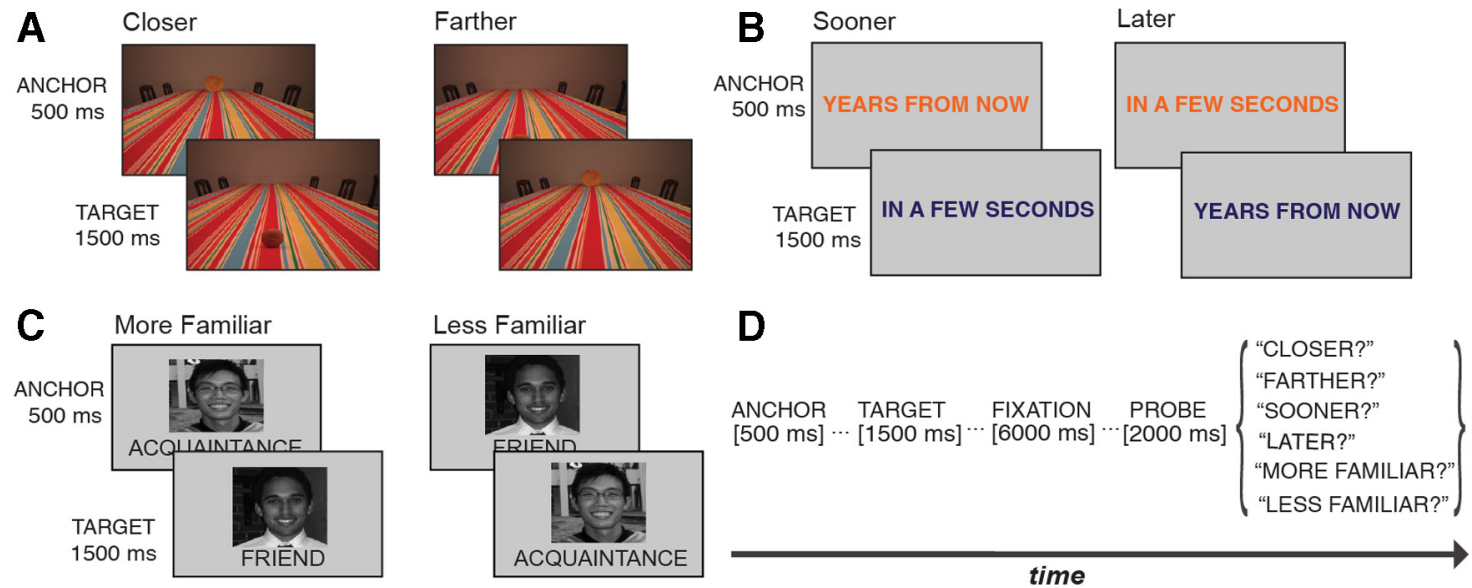

Figure 1. Experimental paradigm. $A-C$, Examples of each trial type. Within each trial, stimulus change over time was analogous to "movement" either toward or away from the observer in a spatial, temporal, or social frame of reference. $\boldsymbol{A}$, Spatial distance trials consisted of household objects photographed at different egocentric distances. $\boldsymbol{B}$, Temporal distance trials consisted of phrases referring to the immediate or more remote future. $C$, Social distance stimuli consisted of the names and photographs of four friends and four acquaintances of each participant. Experimental stimuli contained individuals' actual first and last names rather than the words "friend" and "acquaintance." D, Generic trial schematic. Within each domain, two possible probes varied randomly across trials (e.g., either "Sooner?" or "Later?" for temporal distance trials). Participants responded "Yes" or "No" with a button press. Each trial was followed by an additional $6 \mathrm{~s}$ of fixation before the beginning of the next trial.

Both a shared encoding mechanism and nearby but distinct codes would yield overlapping results (Peelen and Downing, 2007).

To determine whether different domains of egocentric distance are represented by a common code, we used informationbased searchlight mapping (Kriegeskorte et al., 2006), which provides a data-driven method of continuously mapping local information content throughout the brain using multivoxel pattern analysis (MVPA; Norman et al., 2006). Cross-domain searchlights tested whether decision boundaries that distinguish local response patterns based on distance within one domain generalize to other domains. Additionally, we combined representational similarity analysis (RSA; Kriegeskorte et al., 2008) with searchlight mapping to search for regions whose representational content reflect subjective ratings of psychological distance.

\section{Materials and Methods}

Participants. Fifteen participants (10 females; aged 20-28 years; mean age, 24.6 years) completed the study. All participants were right-handed, fluent in English, and had normal or corrected-to-normal vision. Participants provided informed consent, and the experiment was approved by the Dartmouth College Committee for the Protection of Human Subjects.

fMRI paradigm. While being scanned in a 3 T Philips scanner, participants saw spatial, temporal, and social distance trials, consisting of objects photographed at different egocentric distances (Berryhill and Olson, 2009; Fig. 1A), words referring to the immediate or more remote future (Fig. $1 B$ ), and names and photographs of familiar others and acquaintances (Fig. 1C), respectively. To make distances meaningfully comparable across domains, participants were asked to compare each stimulus with a reference point or anchor from the same distance domain. More specifically, each trial began with a briefly presented (500 $\mathrm{ms}$ ) anchor from the same stimulus domain as a target stimulus that was presented for the majority of the trial $(1500 \mathrm{~ms})$. Participants were asked to think about how much closer or farther, more or less familiar, or sooner or later the target was relative to the anchor during spatial, social, and temporal distance trials, respectively. Different trial types within a domain (e.g., closer and farther spatial distance trials) contained the same stimuli in reverse order such that the direction (i.e., either toward or away from the self) of relative egocentric distance changes over time, but not the magnitude of these changes (e.g., $250 \mathrm{~cm}$ for spatial distance trials), differed between trial types (e.g., closer vs farther) within each distance domain (e.g., spatial distance). Thus, in effect, the progression of stimuli over time within each trial was analogous to "movement" by an equivalent amount either toward or away from the participant in a spatial, temporal, or social frame of reference.

Trials were presented according to a slow event-related design. Each trial was followed by $6 \mathrm{~s}$ of fixation and then a probe (e.g., "Later?") that was intended to ensure that participants were comparing the anchor and target stimuli in the intended manner (i.e., in terms of relative egocentric distance and not other aspects of the stimuli). Participants responded "Yes" or "No" to the probe with a Lumina response pad regarding, in this example ("Later?"), whether the target phrase referred to a point in time later in the future than the anchor phrase. All probes were presented in black 96-point font. Each probe was followed by an additional $6 \mathrm{~s}$ of fixation before the beginning of the next trial. Participants used the left response button to indicate "Yes" and the right response button to indicate "No." Within each domain, there were two possible probes that could follow each trial (i.e., either "Sooner?" or "Later?" for temporal distance trials; either "More familiar?" or "Less familiar?" for social distance trials; either "Closer?" or "Farther?" for spatial distance trials). Probes were randomized across trials so that participants could not anticipate the correct response or formulate an appropriate motor plan until after each trial had ended. More specifically, for trials from each distance domain, because participants did not know which of the two possible probes would appear until several seconds after the trial had ended, they could not anticipate the correct button response (i.e., a left button press for "Yes" or a right button press for "No") during trials. This approach also ensured that left and right response buttons were equally likely to signify relatively near and far egocentric distances within each tested distance domain throughout the experiment.

Each participant's stimulus set consisted of pictures of four friends, four acquaintances, four relatively close objects and four relatively distant objects, as well as four verbal phrases referring to the immediate future and four verbal phrases referring to the more remote future. All four friend pictures preceded and followed all four acquaintance pictures an equal number of times during social distance trials, all four photographs of close objects preceded and followed all four photographs of distant objects an equal number of times during spatial distance trials, and all four phrases referring to the immediate future preceded and followed all four phrases referring to the remote future an equal number of times during temporal distance trials. This yielded a total of 96 unique trials per participant. Each run consisted of 36 trials (six instances of each of six trial types: closer, farther, sooner, later, more familiar, less familiar). Within runs, trials were presented in a pseudorandom order to 
approximate second-order counterbalancing of trial type (i.e., closer, farther, sooner, later, more familiar or less familiar). Counterbalanced trial type orders were filled with specific trials by drawing randomly without replacement from lists of all trials belonging to each trial type, with each trial repeated three times. Thus, each unique trial was repeated three times over the course of eight functional runs.

Spatial distance stimuli. All stimuli and probes were presented against a light gray $1280 \times 600$ pixel background (Fig. $1 A$ ). Spatial distance stimuli came from a preexisting stimulus set depicting common household objects either 50 or $300 \mathrm{~cm}$ away amid a naturalistic background containing strong monocular depth cues (Berryhill and Olson, 2009). These stimuli were tested previously to ensure that viewers perceive them to be at the intended egocentric distances and have been demonstrated previously to automatically elicit egocentric distance processing (Berryhill and Olson, 2009). Retinal subtense was approximately consistent across near and far pictures, because stimuli were drawn from four image pairs that depicted similar objects of different sizes (e.g., circular breads: a mini pita and a pizza shell), with the larger and smaller objects photographed at distances of 300 and $50 \mathrm{~cm}$, respectively. The images used in the current study depicted spoons, round breads, beverage pitchers, and round yellow fruits.

Temporal distance stimuli. Temporal distance stimuli consisted of verbal phrases referring to the immediate or more remote future. Previous work suggests that words describing relatively proximal and distal temporal entities are automatically processed in terms of egocentric psychological distance (Bar-Anan et al., 2006, 2007). To ensure readability of these quickly transitioning stimuli, the anchor and target phrases were presented in different font colors: the anchor phrase was always presented in orange text, and the target phrase was always presented in blue text (Fig. 1B). Phrases were presented in 96-point font. Remote future phrases were: "A YEAR FROM NOW"; "DECADES FROM NOW"; "A MONTH FROM NOW"; "IN A FEW YEARS." Immediate future phrases were: "IN A FEW SECONDS"; "MOMENTS FROM NOW"; "IN AN INSTANT"; "A MINUTE FROM NOW." Wilcoxon's rank-sum tests (function wilcox.test in R; R Core Team, 2013) indicated that word counts in immediate (median, 3.50) and remote (median, 4.00) future phrases did not significantly differ $(W=10, p=0.61, r=0.18)$. Letter counts also did not significantly differ between immediate (median, 13.50) and remote (median, 12.50) future phrases $(W=6, p=0.65, r=$ 0.16 ). Additionally, the number of plural nouns was exactly matched between the two trial types (i.e., exactly two of the immediate future phrases and two of the remote future phrases contained one plural noun). The frequency of all phrases and their constituent words were obtained from the Corpus of Contemporary American English (Davies, 2010), a 450 million-word corpus of spoken English, fiction, newspapers, popular magazines, and academic journals from 1990 to 2012. The frequencies of words used in the immediate (median, 1,106,896) and remote (median, 718,023$)$ future phrases did not significantly differ $(W=$ $116.5, p=0.61, r=0.09)$, nor did the frequencies of the exact immediate (median, 102) and remote (median, 291.5) future phrases used ( $W=10$, $p=0.69, r=0.14)$.

Social distance stimuli. Before scanning, participants provided names and front-facing photographs of four people whom they know well and with whom they have a strong personal relationship, and of four people whom they know well but with whom they do not have a strong friendship or interpersonal connection. Familiar and unfamiliar others were matched for gender, race, age, and facial expression (which was either neutral or slightly positive). These photographs were cropped to include only the shoulders, neck, and head and were grayscaled. Photographs were presented at a size of $400 \times 400$ pixels directly above the individual's full name in black 72-point font (Fig. 1C).

Postscan questionnaire. After scanning, 12 participants completed a paper-and-pencil questionnaire consisting of behavioral ratings of psychological distance between stimuli. Stimuli were grouped by domain (social, spatial, temporal); domain order was counterbalanced across participants. All stimulus pairs were presented with the psychologically "closer" stimulus on the left of the psychologically "farther" stimulus. Participants rated the difference in psychological distance between the two stimuli on a 7-point scale between 1 (equally soon/close/familiar) and 7 (the image/phrase on the left is a great deal sooner/closer/more familiar). Distance ratings for trials containing the same stimulus pairs in reverse order were assigned the same numerical ratings multiplied by -1 . One participant completed both this questionnaire and a questionnaire with stimuli presented in reverse order that asked for ratings between 1 (equally late/far/familiar) and 7 (the image/phrase on the left is a great deal later/farther/less familiar). Ratings did not differ between the two versions of the questionnaire.

Image acquisition. Participants were scanned at the Dartmouth Brain Imaging Center using a 3 T Philips Achieva Intera scanner with a 32channel head coil. An echo-planar sequence (35 ms TE; $2000 \mathrm{~ms}$ TR; $3.0 \times 30.0 \times 3.0 \mathrm{~mm}$ resolution) was used to acquire functional images, with 291 dynamic scans per run, for a total acquisition time of $582 \mathrm{~s}$ per run. A high-resolution T1-weighted anatomical scan (8200 ms TR; 3.7 $\mathrm{ms}$ TE; $0.938 \times 0.938 \times 1.0 \mathrm{~mm}$ resolution) was acquired at the end of the scanning session. Foam padding was placed around subjects' heads to minimize motion.

Image preprocessing. Functional data were preprocessed, and parameter estimates ( $\beta$ values) were obtained for each of the six trial types in each run using AFNI (Cox, 1996). Data were time shifted to correct for interleaved slice order, and each volume was spatially registered to the volume immediately preceding the high-resolution anatomical scan. Next, data were despiked to remove transient, extreme values in the signal not attributable to biological phenomena and were spatially smoothed using a $4 \mathrm{~mm}$ full-width at half-maximum Gaussian kernel. Each voxel time series was scaled to a mean amplitude of 100. Next, parameter estimates were extracted for each voxel using the general linear model (GLM). Stimulus events were defined as the $2 \mathrm{~s}$ trial period beginning with the onset of the $0.5 \mathrm{~s}$ presentation of the anchor and ending at the conclusion of the $1.5 \mathrm{~s}$ presentation of the target and were convolved with the standard hemodynamic response function. The GLM included six predictors, one for each trial type, and six regressors of no interest (motion parameters), resulting in an estimate of the response of each voxel to each trial type within each run for use in classification analyses. For RSA and univariate analyses, deconvolution was performed on concatenated data from all eight runs. Gray matter segmentation of each participant's anatomical image was performed using FreeSurfer (Fischl et al., 2004). Gray-matter masks were aligned to anatomical images after the alignment of the anatomical images to functional data and then resampled to the resolution of the functional data.

Classification analyses. The spatial variability of response patterns can reveal information that distinguishes between experimental conditions, even in the absence of overall mean activation differences (Peelen and Downing, 2007). Thus, compared with univariate analyses, MVPA can be more sensitive to differences between trial types within domains (e.g., closer vs farther trials). Additionally, MVPA can provide a more stringent test of whether common cortical mechanisms encode egocentric distance across domains, because overlapping univariate contrasts could result from a shared computational process or from the elicitation of overlapping but functionally independent population responses (Peelen and Downing, 2007). Thus, cross-domain distance decoding based on distributed patterns of fMRI responses provides a useful means of testing whether or not response patterns within a given brain region similarly distinguish between relatively near and relatively far egocentric distances across distance domains.

Six spherical searchlights (radius, 3 voxels) were moved throughout each subject's gray matter-masked data that iteratively performed crossdomain distance decoding (Fig. 2A-C) on local fMRI response patterns using PyMVPA (Hanke et al., 2009). At each searchlight center (i.e., at each voxel), a linear support vector machine (SVM) learning algorithm was trained to discriminate local distributed patterns of fMRI responses to trials from one domain (e.g., social distance) in terms of relative psychological distance (e.g., more vs less familiar) within data from seven of the eight functional runs and was tested on local response patterns corresponding to trials from another distance domain (e.g., temporal distance) from the left-out run. In the aforementioned example, classification would be considered correct if sooner and later trials were classified as more familiar and less familiar trials, respectively. For each searchlight, this procedure was repeated eight times using the data of 
each run for testing once. Classification accuracy was averaged across data folds within each searchlight, resulting in a percentage accuracy score at each voxel for each participant for each searchlight. This procedure was repeated using all possible pairwise combinations of distance domains (social, spatial, temporal) to train and test the pattern classifier, yielding six accuracy maps for each participant. Each accuracy map describes how well the decision boundary that best separates response patterns according to relative egocentric distance within one distance domain generalizes to a new distance domain within a $9 \mathrm{~mm}$ sphere centered on each voxel. For example, the accuracy map corresponding to using spatial distance trials as training data and temporal distance trials as testing data describes the proportion of the time that sooner and later trials (i.e., the testing data) fall on the same side of the decision boundary that best separates closer and farther trials (i.e., the training data) as closer trials and farther trials do, respectively (as illustrated in Fig. $2 B, C$ ).

Percent accuracy maps resulting from crossdomain distance decoding analyses were aligned to standard space (Talairach and Tournoux, 1988) and arcsine transformed ( $y=\operatorname{arcsine} \sqrt{ } x$, where $x$ is proportion of classifications that were correct, and $y$ is the result of the transformation) before being tested against chance across participants, because this procedure approximates a normal and homoscedastic distribution given binary data summarized as proportions (e.g., proportion of correct/incorrect classifications; Freeman and Tukey, 1950; Zar, 1996). Each set of accuracy maps was then submitted to a two-tailed one-sample $t$ test against the arcsine transform of $50 \%$ correct across participants. A conjunction analysis of these six $t$ statistic maps was performed using a threshold of $p<0.05$, false discovery rate (FDR; Benjamini and Hochberg, 1995) corrected, for each map to identify regions in which psychological distance could be decoded across all distance domains.

Importantly, the conjunction analysis requires above-chance distance decoding across all pairs of distance domains. It should be emphasized that, for each classification search-

light, predictions were only considered correct if trials characterized by decreasing psychological distance in the test domain (e.g., more familiar trials) were classified as if they were trials characterized by decreasing psychological distance in the training domain (e.g., sooner trials). Although distances characterizing social and spatial stimuli were inherently self-referential (e.g., $250 \mathrm{~cm}$ closer to or farther away from oneself; differences in social familiarity to oneself), temporal distance stimuli could be compared in terms of the durations implied by the words in these phrases without reference to oneself. However, comparing stimuli in terms of magnitude without respect to distance from the self in the here and now would likely often result in erroneous predictions. For instance, more familiar (i.e., increasing familiarity) trials would be classified as if they were later (i.e., increasing temporal "amount") trials and vice versa, and less familiar (i.e., decreasing familiarity) trials would be classified as if they were sooner (i.e., decreasing temporal "amount") trials and vice versa, which would all be considered inaccurate classifications. Thus, comparing "amounts" implied by stimuli without reference to the self would likely engender incorrect cross-domain classifications in many cases. Because the conjunction analysis requires above-chance distance decoding across all pairs of distance domains, this approach should identify areas that contain a domain-general encoding of egocentric distance.

RSA. A representational similarity searchlight (Kriegeskorte et al., 2008) was used to probe for the existence of brain regions in which the similarity structure of local population responses reflected the similarity structure evinced in each participant's postscan ratings of distance across domains (Fig. 3). Following Kriegeskorte et al. (2008), at each searchlight center, a nonparametric test of representational relatedness was performed to evaluate the significance of the correlation between behavioral and neural representational dissimilarity matrices (RDMs). This procedure allows for each participant's cortex to be continuously mapped in terms of the relatedness of representations manifested in local fMRI responses and behavioral ratings. Thus, this approach provides a direct assessment of how well a participant's behavioral responses capture the information contained in the fMRI patterns within each searchlight sphere.

In general, RSA permits the direct, quantitative comparison of data acquired using diverse methods (e.g., fMRI, behavioral questionnaires) in terms of the information that each dataset contains about a set of experimental conditions. This is possible because, unlike voxel response 


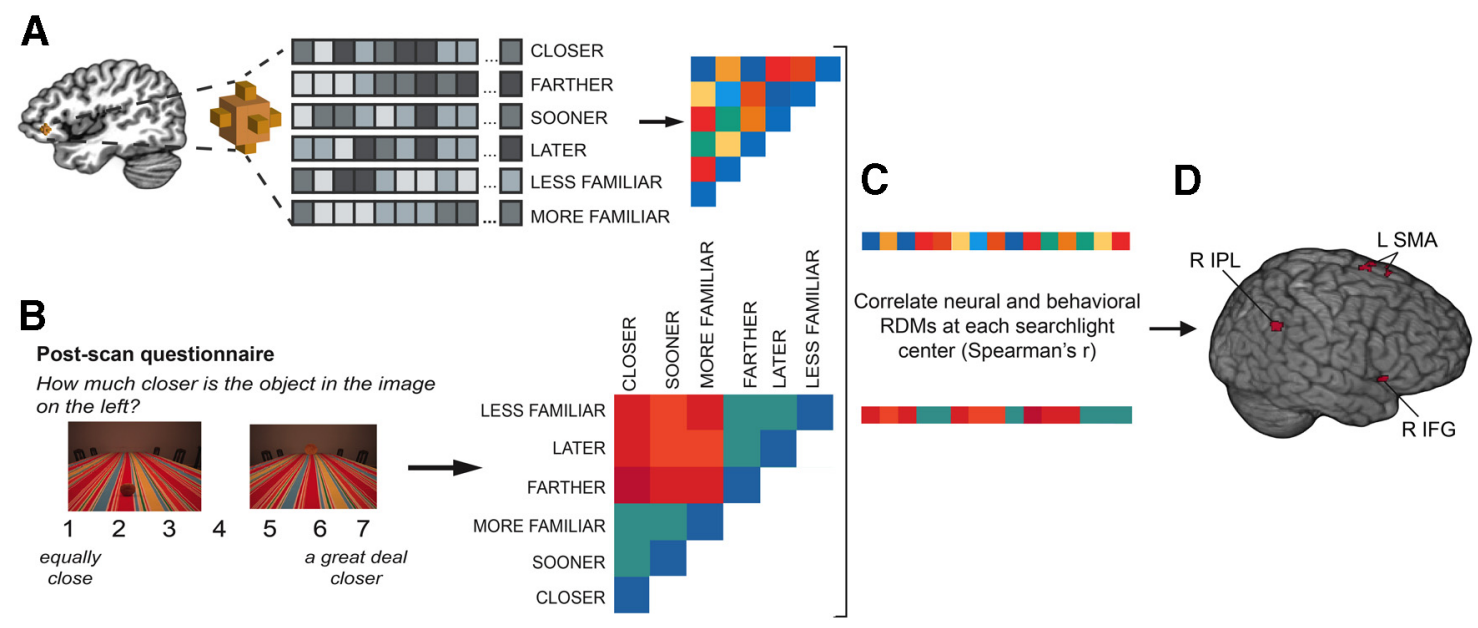

Figure 3. RSA and results. $A-C$, Analysis steps. $A$, At each voxel, a neural RDM was generated based on pairwise correlation distances between local neural response patterns to each condition. Behavioral RDMs were constructed for each subject using Euclidean distances between postscan distance ratings ( $\boldsymbol{B})$ and correlated with local neural RDMs at each voxel (C). $\boldsymbol{D}$, Results. The largest cluster in which local neural and behavioral RDMs were significantly related was in the right IPL (R IPL). Behavioral and neural RDMs were also related in the right IFG (R IFG) and left SMA (LSMA).

patterns, the units of analysis used in RSA (RDMs) are not intrinsically bound to the spatial layout of the original data. RDMs are indexed not by voxel or time point but by experimental condition. Each RDM contains a cell corresponding to the dissimilarity between each pair of experimental conditions (Fig. 4). RSA is uniquely concerned with the relationships between experimental conditions, because its units of analysis (RDMs) only contain information about the dissimilarities between responses to different experimental conditions. An RDM can be constructed that captures the degree to which all pairs of experimental conditions are dissimilar to one another in terms of fMRI responses or behavioral ratings. Thus, it is possible to directly compare the informational content of fMRI and behavioral data by assessing correlations between RDMs generated from fMRI data and those generated from behavioral responses (Kriegeskorte et al., 2008).

Because the current study has six experimental conditions, each neural and behavioral RDM has 15 unique parameters. Thus, RDMs provide a relatively information-rich summary of the information carried in behavioral and fMRI responses. Other methods of relating fMRI data to behavior are often qualitative or involve relating neurometric and psychometric functions, which tend to contain fewer parameters (Kriegeskorte et al., 2008). At the same time, constructing an RDM is a useful dimensionality reduction step, because the number of features in the original fMRI data far exceeds the number of unique elements in each RDM. Thus, after constructing RDMs, data are usefully condensed in comparison with their original form. Additionally, rather than assuming that all distance domains were perceived equivalently, this data analytic approach preserves differences in behavioral ratings between domains. Similarly, because RDMs are generated separately for each participant, idiosyncratic differences in relative psychological distance ratings for each participant are preserved.

RSA was implemented using Python [particularly PyMVPA (Hanke et al., 2009) and SciPy (http://www.scipy.org)]. For each participant, a $6 \times$ 6 behavioral RDM was computed by first averaging behavioral ratings within each trial type and then calculating the Euclidean distances between all possible pairs of these mean trial type ratings (Figs. 3B, 4). Next,
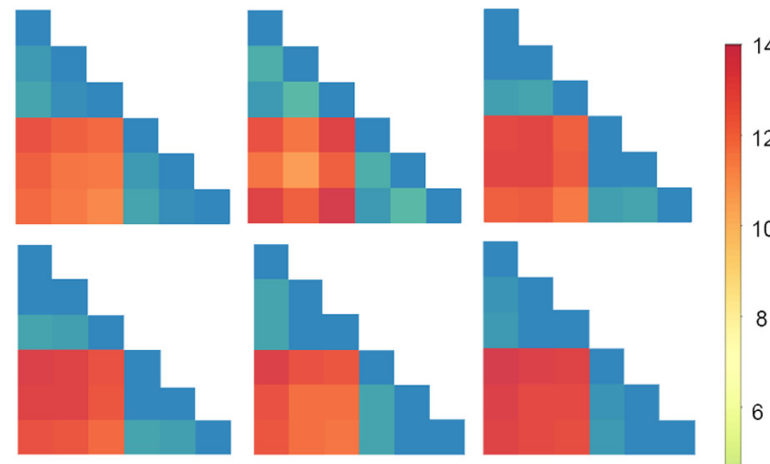

10

8

6
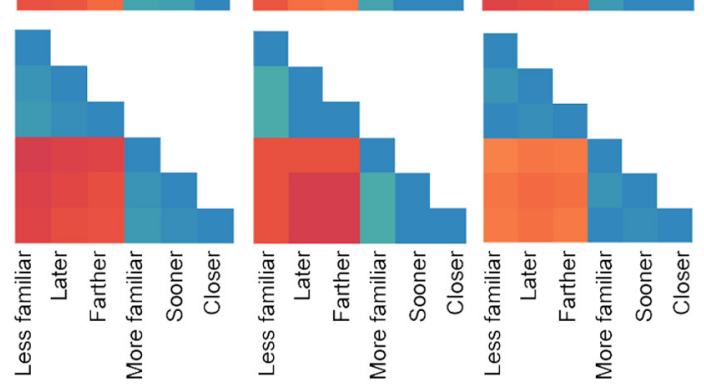

4

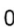

Figure 4. RDMs generated from postscan behavioral distance ratings for each participant. After scanning, 12 participants rated each pair of stimuli seen in the scanner in terms of relative temporal soon-ness, social familiarity, and physical closeness on a 1-7 point scale. The Euclidean distance between each participant's average behavioral rating for each category were used to construct behavioral dissimilarity matrices for his or her similarity searchlight analysis.

a similarity searchlight (radius, 3 voxels) was performed by iteratively computing a neural RDM corresponding to the pairwise Pearson's correlation distances between local multivoxel patterns of parameter estimates corresponding to the six trial types at each voxel in each subject's gray matter-masked data (Fig. 3A). Custom code was written in Python and PyMVPA to compare the off-diagonal elements of the lower triangular halves of the neural and behavioral RDMs at each voxel for each participant (Fig. 3C) using the Spearman's rank coefficient, which was used because it does not assume a linear match between neural and behavioral RDMs (Kriegeskorte et al., 2008). This procedure yielded a statistical map of Spearman's rank coefficients characterizing the relationship between local neural and behavioral RDMs, mapped to the center of each searchlight sphere for each participant.

At each searchlight center, the statistical significance of the relationship between the behavioral and local neural RDMs was evaluated using permutation testing. A distribution of Spearman's rank coefficients cor- 
responding to the null hypothesis that no relationship exists between the neural and behavioral RDMs was obtained by randomly shuffling the domain labels on the behavioral RDM 1000 times and then computing the correlation between the neural RDM and each of the 1000 randomly generated permutations of the behavioral RDM. Next, the inverse of the cumulative normal distribution was calculated to convert the resultant $p$ values into $z$ scores (Connolly et al., 2012). $Z$-statistic maps describing the relationship between the similarity structure of each participant's behavioral ratings of distance for each trial type and the similarity structure of local distributed fMRI response patterns corresponding to each trial type were aligned to standard space (Talairach and Tournoux, 1988) and submitted to a group analysis (one-sample $t$ test against a $z$ score of 0 ) in AFNI (Cox, 1996).

Conventional univariate analyses. A standard univariate analysis using the GLM was performed in AFNI (Cox, 1996) to determine whether the MVPA results merely reflected differences in the overall average magnitude of responses to closer, sooner, and more familiar trials compared with farther, later, and less familiar trials, respectively. Voxelwise parameter estimates generated from concatenated data from all eight runs were submitted to three paired $t$ tests (sooner vs later; closer vs farther; more familiar vs less familiar) using the AFNI program 3 dttest ++ . To determine whether any brain areas could distinguish between closer and farther trials, sooner and later trials, and more familiar and less familiar trials based solely on the overall magnitude of local voxel responses, a conjunction analysis was performed on the statistical maps resulting from each of these three $t$ tests, using a threshold of $p<0.05$, FDR corrected, for each test.

\section{Results}

\section{Behavioral results}

Results of postscan written questionnaires confirmed that participants perceived stimuli pairs from trials in all domains to differ substantially in terms of relative egocentric psychological distance. Mean \pm SD ratings of perceived egocentric distance differences on a scale from 1 (equally soon/close/familiar) to 7 (one image/phrase is a great deal sooner/closer/more familiar) were $6.21 \pm 0.70,6.29 \pm 0.82$, and $6.52 \pm 0.62$ for closer, sooner, and more familiar trials, respectively. One-sample $t$ tests indicated that distance ratings for stimulus pairs from each domain significantly differed from 1 (equally soon/close/familiar; all $p$ values $<0.00001$ ). A one-way ANOVA indicated that perceived relative psychological distance did not significantly differ across the three tested domains of psychological distance $\left(F_{(2,22)}=1.94, p=0.17\right.$, $\eta_{G}^{2}=0.09$ ). Additionally, response accuracies to probes during scanning were high, suggesting that participants were able to attend to and compare the anchors and targets in the intended manner during scanning. Run accuracies ranged from 95.83 to $100 \%($ mean $\pm \mathrm{SD}, 97.97 \pm 1.34 \%)$.

\section{Classification results}

Clusters (cluster size $>10$ voxels) in which relative egocentric distance could be decoded across all possible pairwise combinations of distance domains are presented in Table 1. All voxels in each of these clusters were associated with significantly abovechance classification across participants for each of the six group analyses ( $p<0.05$, FDR corrected, each test). Specifically, relative egocentric distance could be decoded across all distance domains above chance across participants in searchlight spheres centered on voxels in a large (273-voxel) cluster in the right inferior parietal lobule (IPL) extending into the posterior superior temporal gyrus (STG; Fig. 2D). Cross-domain distance decoding was also possible in smaller clusters throughout the right IPL, spanning both the supramarginal (SMG) and angular (AG) gyri, as well as in one cluster in medial occipital cortex, as summarized in Table 1.
Table 1. Regions where relative psychological distance could be decoded from local patterns of voxel responses across all distance domains

\begin{tabular}{llllllll}
\hline Hemisphere & Location & BA & $\begin{array}{l}\text { Number } \\
\text { of voxels }\end{array}$ & $\begin{array}{l}\text { COG } \\
x\end{array}$ & \multicolumn{1}{c}{$\begin{array}{l}\text { COG } \\
\text { COG }\end{array}$} & $\begin{array}{l}\text { Average } \\
\text { peak \% } \\
\text { accuracy }\end{array}$ \\
\hline$R$ & IPL (AG), STG & 39,22 & 273 & 61 & -39 & 9 & 62.33 \\
L, R & LG, (UN & 18 & 200 & 0 & -77 & 5 & 66.12 \\
$R$ & IPL (SMG) & 40 & 70 & 59 & -46 & 21 & 65.18 \\
$R$ & IPL (SMG) & 40 & 29 & 62 & -35 & 31 & 61.30 \\
$R$ & IPL (AG) & 39 & 24 & 54 & -59 & 26 & 62.19 \\
$R$ & IPL (SMG) & 40 & 21 & 60 & -38 & 26 & 66.01 \\
$R$ & STG, IPL (SMG) & 42,40 & 15 & 61 & -28 & 16 & 60.49 \\
\hline
\end{tabular}

All presented results are significant at an FDR-corrected threshold of $p<0.05$ (two-tailed) for each of six statistical tests (corresponding to significance tests for accuracy maps derived from using all possible pairs of the three tested distance domains for training and testing data). To obtain average peak accuracy values, a mask of each of these clusters was applied to average accuracy maps for each of the six tests, and peak percentage accuracies were averaged across tests for each cluster. BA, Brodmann's area; $C O G$, center of gravity; $R$, right; L, left; $L G$, lingual gyrus; CUN, cuneus. All coordinates are in Talairach space.

Table 2. Regions where behavioral and local neural RDMs were significantly correlated

\begin{tabular}{|c|c|c|c|c|c|c|c|c|c|}
\hline Hemisphere & Location & $B A$ & $\begin{array}{l}\text { Number } \\
\text { of voxels }\end{array}$ & $\begin{array}{l}\mathrm{COG} \\
x\end{array}$ & $\begin{array}{l}\mathrm{COG} \\
y\end{array}$ & $\begin{array}{l}C O G \\
z\end{array}$ & $\begin{array}{l}\text { Peak } \\
x\end{array}$ & $\begin{array}{l}\text { Peak } \\
y\end{array}$ & $\begin{array}{l}\text { Peak } \\
z\end{array}$ \\
\hline $\mathrm{R}$ & IPL (AG, SMG) & 39,40 & 113 & 47 & -54 & 36 & 48 & -55 & 36 \\
\hline L & SMA & 6 & 43 & -17 & -3 & 60 & -18 & -2 & 59 \\
\hline$R$ & $\mathbb{I F G}$ & 47 & 35 & 23 & 15 & -11 & 23 & 16 & -11 \\
\hline L & SMA & 6 & 16 & -7 & 13 & 60 & -8 & 12 & 59 \\
\hline L & SMA & 6 & 13 & -17 & 2 & 63 & -17 & 1 & 63 \\
\hline
\end{tabular}

All presented results are significant at an FDR-corrected threshold of $p<0.05$ (two-tailed). BA, Brodmann's area; $C O G$, center of gravity; $R$, right; $L$, left. All coordinates are in Talairach space.

\section{RSA results}

Representational content within a large cluster in the right IPL was significantly related to behavioral distance ratings, suggesting that population codes within searchlights centered in this area contain representations that reflect subjective perceptions of egocentric distance. As summarized in Table 2, the local neural similarity structure in smaller clusters within the right inferior frontal gyrus (IFG) and throughout the left supplementary motor area (SMA) were also significantly related to the similarity structures evinced in participants' behavioral ratings of distance, suggesting that information contained in local population codes within these regions may also be related to judgments of relative egocentric social, spatial, and temporal distance. Additional details regarding all significant clusters $(p<0.05$, FDR corrected; cluster size $>10$ voxels) from this analysis are described in Table 2.

\section{Univariate results}

No voxels survived an FDR-corrected threshold of $p<0.05$ in any of the three $t$ tests comparing trials based on distance within each distance domain (i.e., sooner vs later; more familiar vs less familiar; closer vs farther). Even using a dramatically reduced voxelwise threshold of $p<0.01$, uncorrected, for each test, no regions emerged as significant in the conjunction analysis, suggesting that no regions could distinguish distances in all three distance domains tested here in terms of average activity level. This suggests, at least at the current level of observation, using the current experimental paradigm, that the local distributed patterns, rather than average magnitude, of fMRI responses contain information that distinguishes between different relative egocentric distances across social, spatial, and temporal distance domains. 


\section{Discussion}

The current results suggest that the human brain contains a parsimonious encoding of relative egocentric distance that generalizes to social, spatial, and temporal frames of reference. In several clusters throughout the right IPL, a classifier trained to distinguish trials based on relative egocentric distance within any single domain could distinguish trials within any other domain according to distance above chance across participants. Additionally, the similarity structure of population codes in this region reflected subjective proximity ratings.

What purpose would a domain-general encoding of relative egocentric distance serve? According to construal level theory (CLT; Trope and Liberman, 2010), distance in any domain signifies a common meaning: distance from current first-hand experience. Whereas information relevant to our present experience elicits detailed, concrete representations, humans tend to think about information removed from our current experience using decontextualized, abstract representations (Liberman and Trope, 2008). Recently, Tamir and Mitchell (2011) found fMRI evidence consistent with behavioral findings that different distance domains influence our thoughts and actions analogously. Whereas the current study examined how egocentric distance itself is represented, Tamir and Mitchell (2011) examined how contemplating events from distal or proximal perspectives influences the richness of mental simulations. When participants evaluated their opinions and enjoyment of activities in proximal or distal scenarios, fMRI response magnitudes in areas associated with mental simulation were influenced similarly by different distance domains. Thus, evidence from social psychology and neuroimaging demonstrates analogous effects of different distance domains on cognition and behavior, consistent with suggestions that this information implies a common psychological meaning (Liberman and Trope, 2008). The current results provide evidence for a parsimonious representation of this shared meaning in the brain.

Walsh (2003) suggested that, on the scale of action execution, space, time, and quantity are encoded by a common metric that was repurposed for other magnitudes. Fittingly, areas involved in reaching and eye movements, the horizontal aspect of the intraparietal sulcus (hIPS; Dehaene et al., 2003; Cantlon et al., 2009; Eger et al., 2009) and superior parietal lobule (SPL; Knops et al., 2009), are implicated in representing and processing number. To characterize the contribution of domain-general magnitude representations to psychological distance processing, future studies should examine egocentric distance representations without attempting to minimize magnitude-related effects and directly compare such representations with those of other magnitudes. We predict that representations of magnitudes characterizing egocentric distances would be found in the hIPS, which encodes other magnitudes (e.g., numerosity; Eger et al., 2009); egocentric distances may be encoded similarly to other magnitudes in this region. Conversely, representations more specific to egocentric distance may be found in the right IPL and posterior STG [i.e., the right temporoparietal junction (RTPJ)], in which cross-domain distance decoding was possible in the current study. The RTPJ encodes egocentric space (Schindler and Bartels, 2013) and is thought to support self-other distinctions (Decety and Somerville, 2003). Lesions to this region produce deficits in representing space (Karnath and Rorden, 2012) and events along a mental time line (Saj et al., 2013). The current results suggest that the RTPJ represents egocentric spatial, temporal, and social distances according to a parsimonious coding scheme. Future work should investigate how distance representations in the RTPJ relate to other signals of behavioral relevance and salience (Corbetta et al., 2008). A close relationship between these variables is suggested by mounting behavioral evidence for interactions between motivational relevance and egocentric distance perception (Vagnoni et al., 2012; Cole et al., 2013).

CLT suggests that psychologically proximal information promotes attention to concrete, contextual details, whereas distal information evokes decontextualized, abstract representations (Liberman and Trope, 2008). These modes of cognition are subserved by distinct, competing brain networks: the default mode network $(\mathrm{DMN})$ is associated with internally directed thought involving memory (Buckner and Carroll, 2007; Mason et al., 2007), whereas the dorsal attention network (DAN) supports attention to the external environment (Fox et al., 2005). A third network, the frontoparietal control network (FPCN; Vincent et al., 2008), including the RTPJ (Corbetta et al., 2008), flexibly couples with the DMN and DAN to arbitrate between internally and externally directed cognition (Spreng et al., 2010). The egocentric distance code found here may direct attention to details of the external environment or decontextualized internal representations by influencing connectivity of the FPCN with the DAN and DMN. In the same way that the SPL may encode "movements" along the mental number line and saccades similarly because its connectivity and structure support operations relevant both for computing eye movements and arithmetic (Knops et al., 2009), anatomical characteristics of the RTPJ may render it suitable for representing various kinds of egocentric distance analogously. Future work should test this speculation by comparing network dynamics while participants interact with their current surroundings or mentally traverse psychological distances.

Cross-domain distance decoding was also possible in medial occipital cortex. Because this region is associated with mental imagery during relative spatial distance comparisons (Thompson et al., 2009), it is possible that participants performed analogous mental imagery in social and temporal frames of reference. This result could also arise from communication between areas of an occipitoparietal circuit that integrates visual information into egocentric spatial representations (Kravitz et al., 2011) or topdown modulation of population responses in visual cortex to enhance attention to particular spatial locations, because close temporal and social distances are automatically associated with proximal locations (Bar-Anan et al., 2007).

The largest significant clusters in both classification and similarity analyses were in the right IPL. Smaller clusters in which local information content reflected subjective distance ratings occurred in the left SMA and right IFG. These areas are involved in retrieving spatial locations from memory (Baumann et al., 2010) and may be similarly involved in accessing temporal and social frames of reference. These results may also relate to the relevance of psychological distance to action. Left frontal activity is associated with personal relevance, approach motivation, and attentional narrowing (Harmon-Jones et al., 2006; HarmonJones and Gable, 2009), which are associated with diminished psychological distance (Liberman and Förster, 2009; HarmonJones et al., 2012). Close objects are likely to be personally relevant and elicit immediate action and, thus, may evoke similar SMA activation patterns.

These findings provide preliminary support for speculation that IPL circuitry originally devoted to sensorimotor transformations (Walsh, 2003) and representing one's body in space (Lenggenhager et al., 2006) was "recycled" to operate analogously on increasingly abstract contents as this region expanded during 
evolution (Yamazaki et al., 2009). Such speculations are analogous to cognitive linguists' suggestions that we may speak about abstract relationships in physical terms (e.g., "inner circle") because we think of them in those terms (Lakoff and Johnson, 1980). Consistent with representations of spatial distance scaffolding those of more abstract distances, compelling behavioral evidence demonstrates that task-irrelevant spatial information has an asymmetrically large impact on temporal processing (Casasanto and Boroditsky, 2008; Casasanto et al., 2010; Merritt et al. 2010). Future studies extending this approach to neuroimaging will be instrumental in characterizing relationships between neural representations of different domains of distance.

Interestingly, CMT posits that several aspects of physical experience ("source domains," e.g., elevation) scaffold representations of more abstract concepts ("target domains," e.g., valence). Together, the range of metaphors described by CMT and the present results might suggest that any source and target domains would be represented similarly. We hypothesize that this would be true only to the extent that source and target domains share implications for processing and behavior. Relationships between domains of psychological distance are distinguished from those between other source and target domains because any domain of psychological distance confers a chronically accessible, automatically processed common meaning (Bar-Anan et al., 2006, 2007) that affects perception, cognition, and behavior similarly (Liberman and Trope, 2008). Neural representations of other source and target domains may be less consistently and reciprocally associated (Quadflieg et al., 2011).

In summary, these results provide the first evidence for a common cortical code for relative egocentric spatial, temporal, and social distances. A wealth of behavioral evidence demonstrates that considering information at near or far psychological distances confers a "switch" from concrete, low-level mental construal to more abstracted, decontextualized representations (Liberman and Trope, 2008). The domain-general population code documented here is well situated to provide a mechanism for this switch, because the RTPJ belongs to the FPCN, which can couple with the DMN or DAN to promote internally or externally directed cognition, respectively (Corbetta et al., 2008; Spreng et al., 2010). These findings also support speculation that IPL circuitry originally devoted to spatial computations was recycled to perform analogous operations in increasingly abstract frames of reference (Yamazaki et al., 2009). More generally, the current results are consistent with suggestions that neural mechanisms supporting higher-order cognition may often be best understood in terms of the computations, rather than the domains of knowledge, that they involve (Mitchell, 2008). Although cognition is often studied according to commonsense categories, it would be inefficient for the brain to represent spatial, social, and temporal distances entirely separately if they carry a common psychological meaning, as suggested by strikingly similar effects on predictions, evaluations, and behavior (Liberman and Trope, 2008): proximity to the self in the here and now.

\section{References}

Bar-Anan Y, Liberman N, Trope Y (2006) The association between psychological distance and construal level: evidence from an implicit association test. J Exp Psychol Gen 135:609-622. CrossRef Medline

Bar-Anan Y, Liberman N, Trope Y, Algom D (2007) Automatic processing of psychological distance: evidence from a Stroop task. J Exp Psychol Gen 136:610-622. CrossRef Medline

Baumann O, Chan E, Mattingley JB (2010) Dissociable neural circuits for encoding and retrieval of object locations during active navigation in humans. Neuroimage 49:2816-2825. CrossRef Medline
Benjamini Y, Hochberg Y (1995) Controlling the false discovery rate: a practical and powerful approach to multiple testing. J R Stat Soc Ser B Biol Sci 57:289-300.

Berryhill ME, Olson IR (2009) The representation of object distance: evidence from neuroimaging and neuropsychology. Front Hum Neurosci 3:43. CrossRef Medline

Buckner RL, Carroll DC (2007) Self-projection and the brain. Trends Cogn Sci 11:49-57. CrossRef Medline

Cantlon JF, Platt ML, Brannon EM (2009) Beyond the number domain. Trends Cogn Sci 13:83-91. CrossRef Medline

Casasanto D, Boroditsky L (2008) Time in the mind: using space to think about time. Cognition 106:579-593. CrossRef Medline

Casasanto D, Fotakopoulou O, Boroditsky L (2010) Space and time in the child's mind: evidence for a cross-dimensional asymmetry. Cogn Sci 34: 387-405. CrossRef Medline

Cole S, Balcetis E, Dunning D (2013) Affective signals of threat increase perceived proximity. Psychol Sci 24:34-40. CrossRef Medline

Connolly AC, Guntupalli JS, Gors J, Hanke M, Halchenko YO, Wu YC, Abdi H, Haxby JV (2012) The representation of biological classes in the human brain. J Neurosci 32:2608-2618. CrossRef Medline

Corbetta M, Patel G, Shulman GL (2008) The reorienting system of the human brain: from environment to theory of mind. Neuron 58:306-324. CrossRef Medline

Cox RW (1996) AFNI: software for analysis and visualization of functional magnetic resonance neuroimages. Comput Biomed Res 29:162-173. CrossRef Medline

Davies M (2010) The corpus of contemporary American English as the first reliable monitor corpus of English. Lit Ling Comput 25:447-464. CrossRef

Decety J, Sommerville JA (2003) Shared representations between self and other: a social cognitive neuroscience view. Trends Cogn Sci 7:527-533. CrossRef Medline

Dehaene S, Piazza M, Pinel P, Cohen L (2003) Three parietal circuits for number processing. Cogn Neuropsychol 20:487-506. CrossRef Medline

Eger E, Michel V, Thirion B, Amadon A, Dehaene S, Kleinschmidt A (2009) Deciphering cortical number coding from human brain activity patterns. Curr Biol 19:1608-1615. CrossRef Medline

Eyal T, Liberman N, Trope Y (2008) Judging near and distant virtue and vice. J Exp Soc Psychol 44:1204-1209. CrossRef Medline

Fischl B, van der Kouwe A, Destrieux C, Halgren E, Ségonne F, Salat DH, Busa E, Seidman LJ, Goldstein J, Kennedy D, Caviness V, Makris N, Rosen B, Dale AM (2004) Automatically parcellating the human cerebral cortex. Cereb Cortex 14:11-22. CrossRef Medline

Förster J, Friedman RS, Liberman N (2004) Temporal construal effects on abstract and concrete thinking: consequences for insight and creative cognition. J Pers Soc Psychol 87:177-189. CrossRef Medline

Fox MD, Snyder AZ, Vincent JL, Corbetta M, Van Essen DC, Raichle ME (2005) The human brain is intrinsically organized into dynamic, anticorrelated functional networks. Proc Natl Acad Sci U S A 102:9673-9678. CrossRef Medline

Freeman MF, Tukey JW (1950) Transformations related to the angular and the square root. Ann Math Statist 21:607-611. CrossRef

Friston KJ, Holmes AP, Worsley KJ, Poline JP, Frith CD, Frackowiak RSJ (1995) Statistical parametric maps in functional imaging: a general linear approach. Hum Brain Mapp 2:189-210.

Hanke M, Halchenko YO, Sederberg PB, Hanson SJ, Haxby JV, Pollmann S (2009) PyMVPA: a python toolbox for multivariate pattern analysis of fMRI data. Neuroinformatics 7:37-53. CrossRef Medline

Harmon-Jones E, Gable PA (2009) Neural activity underlying the effect of approach-motivated positive effect on narrowed attention. Psychol Sci 20:406-409. CrossRef Medline

Harmon-Jones E, Lueck L, Fearn M, Harmon-Jones C (2006) The effect of personal relevance and approach-related action expectation on relative left frontal cortical activity. Psychol Sci 17:434-440. CrossRef Medline

Harmon-Jones E, Price TF, Gable PA (2012) The influence of affective states on cognitive broadening/narrowing: considering the importance of motivational intensity. Soc Pers Psychol Comp 6:314-327. CrossRef

Karnath HO, Rorden C (2012) The anatomy of spatial neglect. Neuropsychologia 50:1010-1017. CrossRef Medline

Knops A, Thirion B, Hubbard EM, Michel V, Dehaene S (2009) Recruitment of an area involved in eye movements during mental arithmetic. Science 324:1583-1585. CrossRef Medline 
Kravitz DJ, Saleem KS, Baker CI, Mishkin M (2011) A new neural framework for visuospatial processing. Nat Rev Neurosci 12:217-230. CrossRef Medline

Kriegeskorte N, Goebel R, Bandettini P (2006) Information-based functional brain mapping. Proc Natl Acad Sci U S A 103:3863-3868. CrossRef Medline

Kriegeskorte N, Mur M, Bandettini P (2008) Representational similarity analysis: connecting the branches of systems neuroscience. Front Syst Neurosci 2:4. CrossRef Medline

Lakoff G, Johnson M (1980) Metaphors we live by. Chicago: University of Chicago.

Lenggenhager B, Smith ST, Blanke O (2006) Functional and neural mechanisms of embodiment: importance of the vestibular system and the temporal parietal junction. Rev Neurosci 17:643-657. Medline

Liberman N, Förster J (2009) Distancing from experienced self: How global-versus-local perception affects estimation of psychological distance. J Pers Soc Psychol 97:203-216. CrossRef Medline

Liberman N, Trope Y (2008) The psychology of transcending the here and now. Science 322:1201-1205. CrossRef Medline

Mason MF, Norton MI, Van Horn JD, Wegner DM, Grafton ST, Macrae CN (2007) Wandering minds: the default network and stimulusindependent thought. Science 315:393-395. CrossRef Medline

McGraw AP, Warren C, Williams LE, Leonard B (2012) Too close for comfort, or too far to care? Finding humor in distant tragedies and close mishaps. Psychol Sci 23:1215-1223. CrossRef Medline

Merritt DJ, Casasanto D, Brannon EM (2010) Do monkeys think in metaphors? Representations of space and time in monkeys and humans. Cognition 117:191-202. CrossRef Medline

Mitchell JP (2008) Activity in right temporo-parietal junction is not selective for theory-of-mind. Cereb Cortex 18:262-271. CrossRef Medline

Norman KA, Polyn SM, Detre GJ, Haxby JV (2006) Beyond mind-reading: Multi-voxel pattern analysis of fMRI data. Trends Cogn Sci 10:424-430. CrossRef Medline

Parkinson C, Wheatley T (2013) Old cortex, new contexts: re-purposing spatial perception for social cognition. Front Hum Neurosci 7:645. CrossRef Medline

Peelen MV, Downing PE (2007) Using multi-voxel pattern analysis of fMRI data to interpret overlapping functional activations. Trends Cogn Sci 11:4-5. CrossRef Medline

Quadflieg S, Etzel JA, Gazzola V, Keysers C, Schubert TW, Waiter GD, Macrae CN (2011) Puddles, parties, and professors: linking word categorization to neural patterns of visuospatial coding. J Cogn Neurosci 23:2636-2649. CrossRef Medline
R Core Team (2013) R: a language and environment for statistical computing. Vienna: R Foundation for Statistical Computing.

Saj A, Fuhrman O, Vuilleumier P, Boroditsky L (2013) Patients with left spatial neglect also neglect the "left side" of time. Psychol Sci. Advance online publication. Retrieved January 3, 2014. doi:10.1177/0956797612475222. CrossRef Medline

Schindler A, Bartels A (2013) Parietal cortex codes egocentric space beyond the field of view. Curr Biol 23:177-182. CrossRef Medline

Spreng RN, Mar RA, Kim AS (2009) The common neural basis of autobiographical memory, prospection, navigation, theory of mind, and the default mode: a quantitative meta-analysis. J Cogn Neurosci 21:489-510. CrossRef Medline

Spreng RN, Stevens WD, Chamberlain JP, Gilmore AW, Schacter DL (2010) Default network activity, coupled with the frontoparietal control network, supports goal-directed cognition. Neuroimage 53:303-317. CrossRef Medline

Suddendorf T, Corballis MC (2007) The evolution of foresight: what is mental time travel, and is it unique to humans? Behav Brain Sci 30:299 313; discussion 313-351. CrossRef Medline

Talairach J, Tournoux P (1988) Co-planar stereotaxic atlas of the human brain. New York: Thieme Medical Publishers.

Tamir DI, Mitchell JP (2011) The default network distinguishes construals of proximal versus distal events. J Cogn Neurosci 23:2945-2955. CrossRef Medline

Thompson WL, Slotnick SD, Burrage MS, Kosslyn SM (2009) Two forms of spatial imagery: neuroimaging evidence. Psychol Sci 20:1245-1253. CrossRef Medline

Trope Y, Liberman N (2010) Construal-level theory of psychological distance. Psychol Rev 117:440-463. CrossRef Medline

Vagnoni E, Lourenco SF, Longo MR (2012) Threat modulates perception of looming visual stimuli. Curr Biol 22:R826-R827. CrossRef Medline

Vallacher RR, Wegner DM (1985) A theory of action identification. Hillsdale, NJ: Erlbaum.

Vincent JL, Kahn I, Snyder AZ, Raichle ME, Buckner RL (2008) Evidence for a frontoparietal control system revealed by intrinsic functional connectivity. J Neurophysiol 100:3328-3342. CrossRef Medline

Walsh V (2003) A theory of magnitude: common cortical metrics of time, space and quantity. Trends Cogn Sci 7:483-488. CrossRef Medline

Yamakawa Y, Kanai R, Matsumura M, Naito E (2009) Social distance evaluation in human parietal cortex. PLoS One 4:e4360. CrossRef Medline

Yamazaki Y, Hashimoto T, Iriki A (2009) The posterior parietal cortex and non-spatial cognition. F1000 Biol Rep 1:74. CrossRef Medline

Zar JH (1996) Biostatistical analysis. Upper Saddle River, NJ: Prentice Hall. 